\title{
Dynamics, Stability, and Control of Multivariable Piezoelectric Shunts
}

\author{
S. O. R. Moheimani, Senior Member, IEEE, Andrew J. Fleming, Member, IEEE, and Sam Behrens
}

\begin{abstract}
This paper is concerned with the dynamics and stability of piezoelectric laminate structures, where several piezoelectric elements are shunted by a multiinput impedance. The problem is shown to be equivalent to a multivariable feedback control problem for a square plant. A parameterization of stabilizing admittance transfer function matrices is given together with a specific class of controllers capable of reducing structural vibrations and guaranteeing closed-loop stability. An efficient method for implementation of a multiport admittance transfer function is introduced and applied experimentally to demonstrate the effectiveness of the proposed methodology.
\end{abstract}

Index Terms-Dynamics, multivariable, piezoelctric shunts, stability.

\section{INTRODUCTION}

$\mathbf{P}$ IEZOELECTRIC transducers have been used extensively as actuators, and also as sensors in active control of structural vibrations, where the piezoelectric transducer is either used as a sensor, or as an actuator [1]-[6]. However, due to the nature of the piezoelectric effect, it is possible to combine both functions of sensing and actuation in a single device. This fact has generated increasing interest in passive control of vibrations by shunting piezoelectric transducers with electrical impedances. This process effectively integrates sensing and actuation capabilities within a single piezoelectric transducer. An analysis of this procedure is given in [7], where the authors suggest that a piezoelectric transducer can be shunted by a series combination of a resistor and an inductor. The piezoelectric transducer is modeled as a strain-dependent voltage source in series with a capacitor. The resulting $R L C$ circuit is tuned to one of the resonance frequencies of the base structure to suppress structural vibrations due to that specific mode.

The method suggested in [7], although effective, can only be applied to one vibration mode. However, following [7], a number of authors attempted to extend this technique to allow for passive damping of several modes. In [8], the author proposed the use of current blocking circuits to separate $R L$ branches tuned to each resonance frequency. The method works well for a small number of modes. However, as the number of modes increases so does the complexity of the electric shunt, resulting in implementation difficulties.

Reference [9] suggested parallel combination of a series RL circuit with several series $R L C$ branches. The author experimen-

Manuscript received July 17, 2002; revised June 17, 2003. This work was supported by the Australian Research Council.

The authors are with the School of Electrical Engineering and Computer Science, The University of Newcastle, Newcastle, Australia (e-mail: reza@ee.newcastle.edu.au).

Digital Object Identifier 10.1109/TMECH.2004.823882 tally demonstrated the effectiveness of this specific structure in reducing vibrations due to two modes of a structure. However, the synthesis procedure is not straightforward, making it difficult to extend the application to more modes.

The use of parallel combination of series RLC branches was studied in [10]. The idea is to introduce "current flowing" $R C$ circuits in each $R L$ branch. Complexity of the electrical shunt proposed in [10] is considerably less than that proposed in [8]. However, the freedom in choice of the capacitive, or alternatively the inductive elements may complicate the design process.

Shunt damping of piezoelectric laminate structures is currently an active area of research in which new applications are emerging. For some interesting applications, the reader is referred to [11]-[17] and references therein.

This paper is concerned with the problem of multimode shunt damping of structural vibrations using several piezoelectric transducers. It is argued that the problem can be cast as a multivariable feedback control problem, in which the impedance, or alternatively the admittance of the electrical shunt, constitutes the feedback controller. The model of a flexible structure is made up of a large number of highly resonant modes. In a typical vibration-control application, often, only a limited number of low-frequency modes are to be controlled. In this paper, conditions under which the closed-loop system remains stable in the presence of uncontrolled out-of-bandwidth modes are derived, and a number of specific structures for the electrical shunt are proposed.

The main difficulty associated with implementing piezoelectric shunt impedances is the need for implementing very large inductors - in the order of several hundred to several thousand henrys. Synthetic inductors constructed from opamps [18] have been proposed as a possible solution. However, if a large number of modes are to be controlled, one may need to construct an electronic circuit comprising of many opamps. Such circuits could be sensitive to operating conditions and may have to be tuned on a regular basis.

The synthetic admittance circuit proposed in [19] is a viable solution to the above problem. It enables one to implement any given admittance transfer function in an efficient way, as demonstrated in [19]. Furthermore, by using an array of such circuits a multiinput admittance transfer function, of the kind proposed in this paper, can be implemented.

The use of synthetic inductors, or synthetic admittance systems is probably the only possible means of implementing electric shunts onto piezoelectric transducers. Strictly speaking, the resulting impedance will not be a "passive" circuit, as it will be made of "active" components, such as opamps and transistors, 
digital signal processing (DSP) chips, etc. However, there are clear advantages in using such techniques. Shunt damping circuits remove the need for external sensors since the piezoelectric transducers act as sensors as well as actuators. Furthermore, the feedback structure associated with the shunt damping systems indirectly recovers the collocated transfer function of the system in an indirect way (see Section II). The latter is particularly beneficial in terms of constructing impedance structures that guarantee closed-loop stability in presence of out-of-bandwidth dynamics.

The remainder of the paper continues as follows. Section II is concerned with the feedback structure associated with shunt damping systems with multiple piezoelectric transducers. Section III presents a parameterization of stabilizing impedances for such systems. Section IV introduces a class of decentralized admittance transfer functions that apart from being stabilizing are very efficient in reducing structural vibrations of the base structure. Section V includes our experimental results, and Section VI concludes the paper.

\section{Dynamics of Shunted Piezoelectric Laminate STRUCTURE}

Consider a flexible structure with $m$ piezoelectric patches bonded to its either side in a collocated pattern. Furthermore, assume that the piezoelectric transducers on one side are used to disturb the structure by generating vibrations, while those on the other side of the structure are shunted to an impedance. The impedance is to be designed in a way that the unwanted structural vibrations are minimized. It should be noted that the disturbances acting on the structure can take different forms. Nevertheless, the methodology developed in the sequel is general enough to apply to such cases. This point will be further clarified in due course.

In Fig. 1(a), a schematic of this system is depicted while the equivalent electrical circuit of the shunted piezoelectric transducers are drawn in Fig. 1(b). Notice that each shunted piezoelectric transducer is modeled as a dependent voltage source in series with a capacitor. Each voltage $v_{p_{k}}$ is proportional to mechanical strain, which is in turn related to the disturbances acting on the structure. Furthermore, $C_{p_{k}}$ represents the capacitance of the $i$ th piezoelectric patch. Dynamics of the shunted system are derived next.

Let

$$
\begin{array}{cc}
V_{z}(s)=\left[\begin{array}{c}
v_{z_{1}}(s) \\
v_{z_{2}}(s) \\
\vdots \\
v_{z_{m}}(s)
\end{array}\right] \quad V_{p}(s)=\left[\begin{array}{c}
v_{p_{1}}(s) \\
v_{p_{2}}(s) \\
\vdots \\
v_{p_{m}}(s)
\end{array}\right] \\
V_{\mathrm{in}}(s)=\left[\begin{array}{c}
v_{\mathrm{in}_{1}}(s) \\
v_{\mathrm{in}_{2}}(s) \\
\vdots \\
v_{\mathrm{in}_{m}}(s)
\end{array}\right] \quad I_{z}(s)=\left[\begin{array}{c}
i_{1}(s) \\
i_{2}(s) \\
\vdots \\
i_{m}(s)
\end{array}\right] .
\end{array}
$$

Then

$$
V_{z}(s)=Z(s) I_{z}(s) .
$$

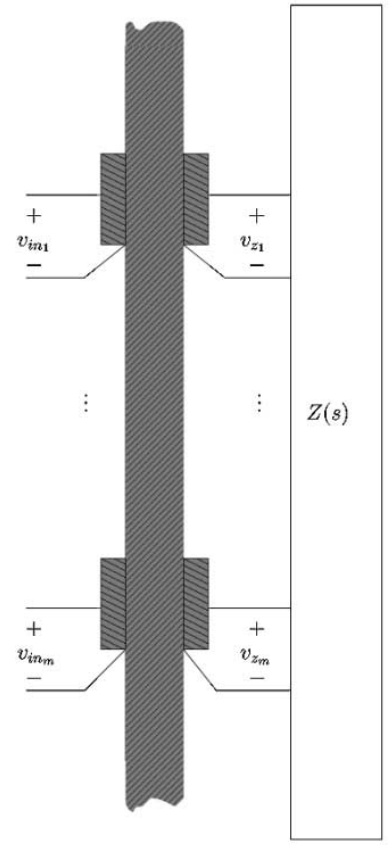

(a)

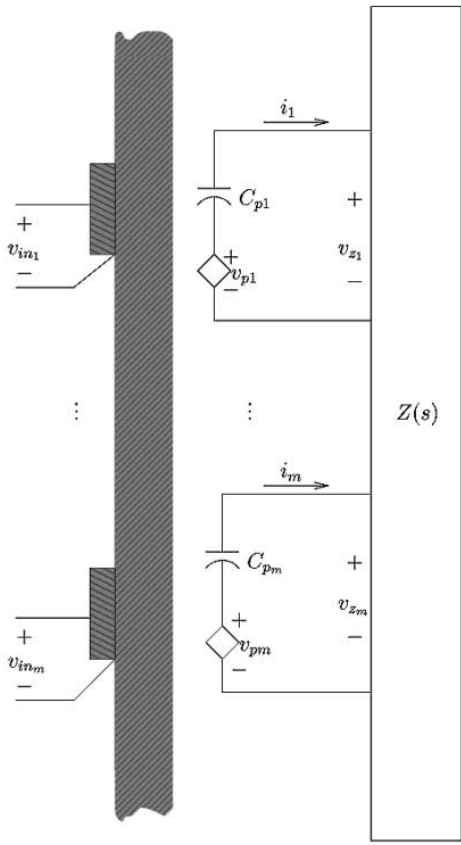

(b)
Fig. 1. (a) Piezoelectric laminate structure with $m$ shunted piezoelectric patches. (b) Electrical equivalent of part (a).

Furthermore, writing the Kirchhoff's voltage law (KVL) around the $k$ th loop we obtain

$$
v_{z_{k}}=v_{p_{k}}-\frac{1}{C_{p_{k}} s} i_{k}
$$

which implies

$$
V_{z}(s)=V_{p}(s)-\frac{1}{s} \Lambda I_{z}(s)
$$

where

$$
\Lambda=\operatorname{diag}\left(\frac{1}{C_{p_{1}}}, \frac{1}{C_{p_{2}}}, \ldots, \frac{1}{C_{p_{m}}}\right)
$$

and $\operatorname{diag}\left(\alpha_{1}, \alpha_{2}, \ldots, \alpha_{m}\right)$ represents a matrix whose diagonal entries are $\alpha_{1}, \alpha_{2}, \ldots, \alpha_{m}$ and whose all other entries are zeros.

To capture the total effect of the disturbance voltages as well as the effect of the electric shunt on the structure, we may write [7]

$$
V_{p}(s)=G_{v v}(s) V_{\text {in }}(s)-G_{v v}(s) V_{z}(s) .
$$

Here, $G_{v v}(s)$ is the multivariable collocated transfer function matrix of the system; i.e.,

$$
G_{v v}(s)=\sum_{k=1}^{M} \frac{\Psi_{k}}{s^{2}+2 \zeta_{k} \omega_{k} s+\omega_{k}^{2}}
$$

where resonance frequencies are ordered such that $\omega_{1} \leq \omega_{2} \leq$ $\cdots \leq \omega_{M}$ and $M$ can be an arbitrarily large number. Furthermore, due to the fact that $G_{v v}(s)$ is a collocated transfer function matrix, the $m \times m$ matrix $\Psi_{k}$ must be a positive semi-definite matrix [20]. That is

$$
\Psi_{k}=\Psi_{k}^{\prime} \geq 0 \quad \text { for all } k .
$$




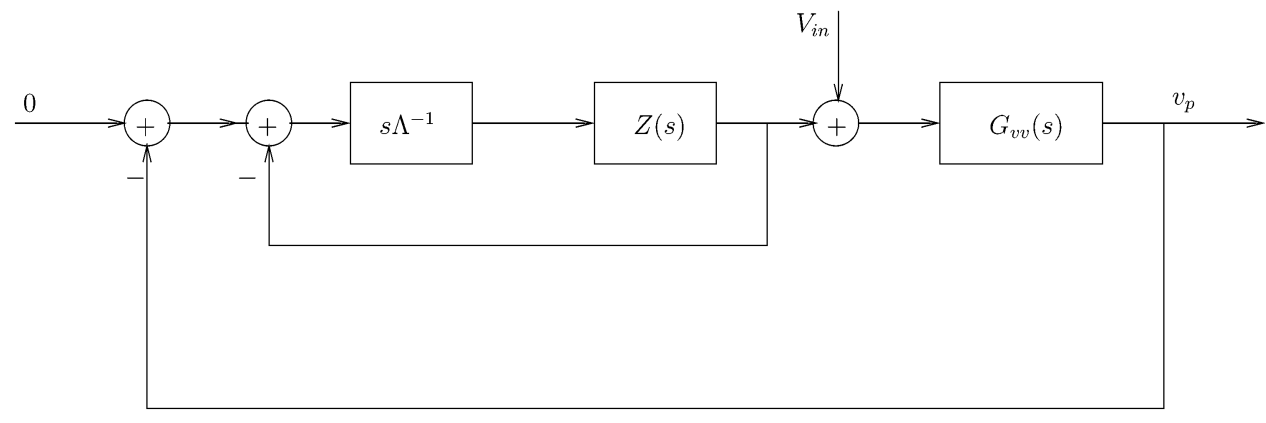

Fig. 2. Feedback structure associated with the shunt damping problem.

It should be pointed out that if (5) is obtained by employing a procedure such as modal analysis [21], one would expect $M \longrightarrow \infty$. However, choosing a very large number for $M$ is quite acceptable, as pointed out in [22]. This would enable us to use finite-dimensional techniques in analyzing dynamics of the system. Models of the form (5) can be obtained using a variety of techniques; e.g., modal analysis if the system is simple with well-defined boundary conditions, or finite element method (FEM) for more complicated structures. An alternative approach is to employ frequency-domain system-identification techniques [23] to identify a model for the system. Frequency-domain subspace identification has proved to be an efficient method for identifying highly resonant systems of high orders [24].

Next, (1), (2), and (4) are combined to obtain

$$
\begin{aligned}
V_{p}(s)=\left[I+G_{v v}(s) Z(s)\left(Z(s)+\frac{1}{s} \Lambda\right)^{-1}\right]^{-1} & \\
& \times G_{v v}(s) V_{\text {in }}(s) .
\end{aligned}
$$

From (7) it can be inferred that the transfer function matrix relating $V_{\text {in }}(s)$ to $V_{p}(s)$ is the feedback connection of $G_{v v}(s)$ with

$$
K(s)=Z(s)\left(Z(s)+\frac{1}{s} \Lambda\right)^{-1}
$$

This is an interesting observation that enables us to employ systems theoretic tools in analyzing dynamics and stability of shunt-damped systems. The feedback control problem associated with (7) is depicted in Fig. 2. Note that the inner feedback loop represents the effective controller $K(s)$ in (8). Observe that the purpose of the system is to regulate $v_{p}$ in the presence of disturbance $V_{\mathrm{in}}$. The signal $v_{p}$, however, is not directly measurable. The reader may notice that this is a very specific form of cascade feedback control structure [25, Sec. VI-D ].

The above system is mainly used in laboratory experiments. Indeed, experimental results of this paper are obtained from a simply-supported beam with two pairs of collocated piezoelectric transducers (see Section V). In a more realistic setting, the disturbances acting on the structure have a different nature. For example, they may be point forces, moments, a distributed force, etc., In this situation, (4) should be modified to

$$
V_{p}(s)=G_{v w}(s) W(s)-G_{v v}(s) V_{z}(s)
$$

where $G_{v w}(s)$ is the unshunted transfer function from the disturbance vector $W(s)$ to $V_{p}(s)$. The transfer function $G_{v w}(s)$ depends solely on the nature as well as the spatial coordinates of the disturbance signal $w$. Nonetheless, due to the common-pole property of flexible structures, $G_{v w}(s)$ and $G_{v v}(s)$ will have identical poles. The zeros of the two transfer functions, however, could be quite different.

An implication of (9) is that the shunted structural dynamics will have to be revised as

$$
\begin{aligned}
V_{p}(s)=\left[I+G_{v v}(s) Z(s)\left(Z(s)+\frac{1}{s} \Lambda\right)^{-1}\right]^{-1} \\
\times G_{v w}(s) W(s) .
\end{aligned}
$$

Observe that although the nature of the disturbance has changed, stability of the shunted system is still dictated by the feedback connection of $G_{v v}(s)$ and $K(s)$ in (8). Furthermore, it is noted that under these circumstances, the regulator problem depicted in Fig. 2 should be modified to that shown in Fig. 3.

\section{StABility OF THE SHUNTED System}

A set of conditions under which stability of the closed-loop system depicted in Fig. 3 is guaranteed, is derived in this section. Instead of considering the shunting impedance $Z(s)$ as the controller, the closed-loop stability of the system is studied in terms of the shunted admittance $Y(s)=Z(s)^{-1}$, noting that the closed-loop transfer function in (10) can be rewritten as

$$
V_{p}(s)=\left[I+G_{v v}(s)\left(I+\frac{1}{s} \Lambda Y(s)\right)^{-1}\right]^{-1} G_{v w}(s) W(s) .
$$

The regulator problem associated with this system is depicted in Fig. 4. A parameterization of stabilizing controllers for the system in (11) is introduced next.

Considering the structure of the feedback system, the Youla parameterization [26] of all stabilizing controllers for the inner feedback loop can be written as

$$
Y(s)=(I-Q(s) \Lambda / s)^{-1} Q(s) .
$$

Although the inner loop contains integrators, the parameterization for a stable plant can be used as long as $Q(s)$ satisfies a number of conditions. Namely, $Q(s)$ must be stable, proper 


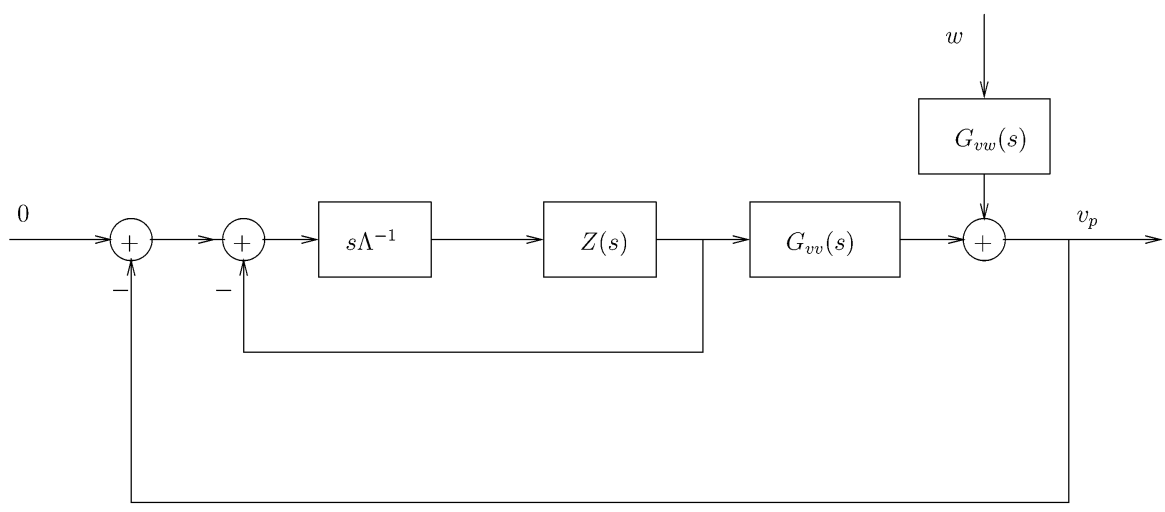

Fig. 3. Feedback structure associated with the modified shunt damping problem.

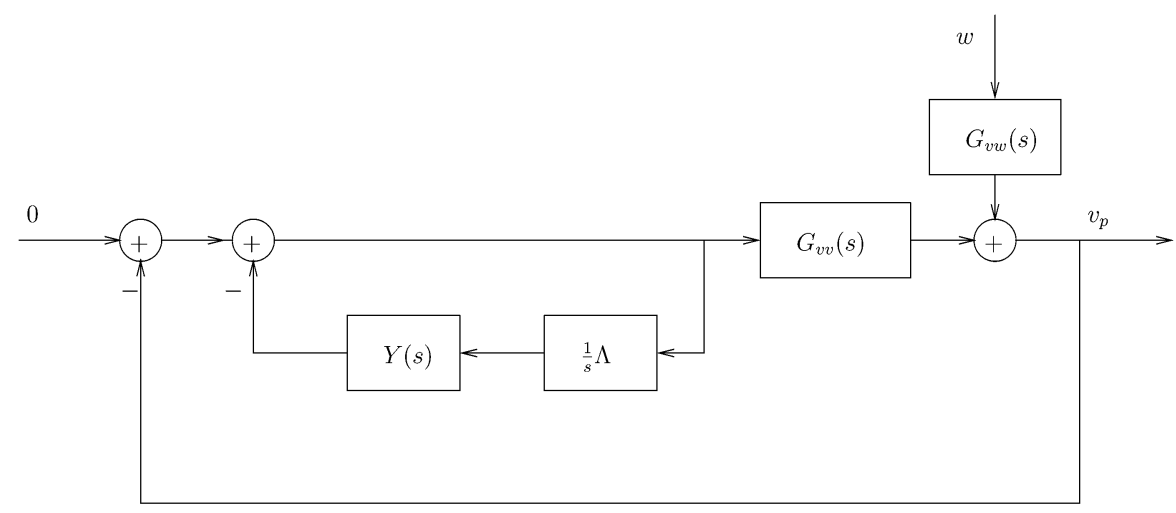

Fig. 4. Feedback structure associated with the shunt damping problem with admittance as the control variable.

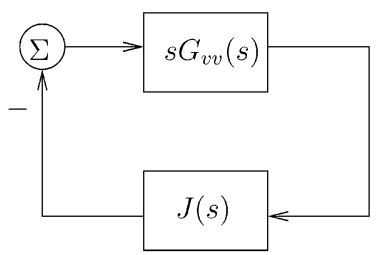

Fig. 5. Feedback connection of $s G_{v v}(s)$ with $J(s)$.

and have a transmission zero at the origin. Furthermore, $I-$ $Q(s) \Lambda / s$ must have a transmission zero at $s=0$. These conditions can be enforced by choosing

$$
Q(s)=H(s) \Lambda^{-1} s
$$

where $H(s)$ is stable, strictly proper, and $I-H(s)$ has a transmission zero at the origin, i.e.,

$$
I-H(s)=s J(s) .
$$

This choice for $Q(s)$ results in a closed-loop system with the transfer function matrix

$$
\left[I+s G_{v v}(s) J(s)\right]^{-1} G_{v w}(s) .
$$

It is now possible to find closed-loop stability conditions in terms of $J(s)$ as the stability of (12) is equivalent to that of the system depicted in Fig. 5.
It turns out that the closed-loop system will be stable as long as $J(s)$ is a strictly positive real (SPR) transfer function matrix. The following two definitions and the subsequent theorem due to [27] are needed in the proofs.

Definition 1: An $m \times m$ rational matrix $G(s)$ is said to be positive real (PR) if

1) all elements of $G(s)$ are analytic in $\operatorname{Re}(s)>0$;

2) $G(s)+G^{*}(s) \geq 0$ in $\operatorname{Re}(s)>0$ or equivalently

a) poles on the imaginary axis are simple and have nonnegative residues;

b) $G(j \omega)+G^{*}(j \omega) \geq 0$ for $\omega \in(-\infty, \infty)$.

Definition 2: An $m \times m$ stable rational matrix $G(s)$ is said to be SPR in the weak sense (WSPR) if

$$
G(j \omega)+G^{*}(j \omega)>0, \quad \text { for } \omega \in(-\infty, \infty) .
$$

The following theorem is Corollary 1.1 of [27].

Theorem 1: The negative feedback connection of a PR system with a WSPR controller is stable.

It should be pointed out that there are a number of definitions in the literature for SPR systems. For a comprehensive review of these, the reader is referred to [28], [27]. For almost all such definitions, one would expect a similar result to that of Theorem 1 ; i.e., the negative feedback connection of a PR system with an SPR controller is stable. It turns out that for the problem at hand, definition 2 is the most relevant.

Now, we prove that

$$
\tilde{G}_{v v}(s)=s G_{v v}(s)
$$


is a PR transfer function matrix. It can be noticed from (13) and (5), that all of the poles of $\tilde{G}_{v v}(s)$ are in the left half of the complex plane; hence, the system is stable. Furthermore, the system has no poles on the $j \omega$ axis. To prove positive realness of $\tilde{G}_{v v}(s)$, we need to establish that $\tilde{G}_{v v}(j \omega)+\tilde{G}_{v v}^{*}(j \omega) \geq 0$ for all $\omega \in(-\infty, \infty)$.

$$
\begin{aligned}
& \tilde{G}_{v v}(j \omega)+\tilde{G}_{v v}^{*}(j \omega) \\
& \quad=\sum_{k=1}^{N}\left\{\frac{j \omega \Psi_{k}}{\omega_{k}^{2}-\omega^{2}+j 2 \zeta_{k} \omega_{k} \omega}\right. \\
& \left.+\frac{-j \omega \Psi_{k}}{\omega_{k}^{2}-\omega^{2}+-j 2 \zeta_{k} \omega_{k} \omega}\right\} \\
& \quad=\sum_{k=1}^{N} \frac{4 \zeta_{k} \omega_{k} \omega^{2} \Psi_{k}}{\left(\omega_{k}^{2}-\omega^{2}\right)^{2}+\left(2 \zeta_{k} \omega_{k} \omega\right)^{2}} \\
& \quad \geq 0 \text { for all } \omega \in(-\infty, \infty)
\end{aligned}
$$

where the last inequality follows from (6).

An implication of the above analysis is that to guarantee the closed-loop stability of the system, it would suffice to choose an admittance

$$
Y(s)=J(s)^{-1}(I-s J(s)) \Lambda^{-1}
$$

with $J(s)$ an WSPR transfer function matrix.

\section{Decentralized ImPEDANCe Design}

The observation made in Section III enables us to design impedance structures that guarantee closed-loop stability of the shunted system. This section introduces two specific decentralized structures that enforce the above conditions. Furthermore, these decentralized impedances result in effective wideband reduction of vibrations of the base structure.

These admittances are constructed starting from

$$
\begin{aligned}
J_{a}(s)= & \sum_{i=1}^{N} \operatorname{diag}\left(\frac{\alpha_{1 i}\left(s+2 d_{1 i} \omega_{i}\right)}{s^{2}+2 d_{1 i} \omega_{i} s+\omega_{i}^{2}}\right. \\
& \frac{\alpha_{2 i}\left(s+2 d_{2 i} \omega_{i}\right)}{s^{2}+2 d_{2 i} \omega_{i} s+\omega_{i}^{2}}, \ldots, \\
& \left.\frac{\alpha_{m i}\left(s+2 d_{m i} \omega_{i}\right)}{s^{2}+2 d_{m i} \omega_{i} s+\omega_{i}^{2}}\right)
\end{aligned}
$$

and

$$
\begin{aligned}
J_{b}(s)= & \sum_{i=1}^{N} \operatorname{diag}\left(\frac{\alpha_{1 i} s}{s^{2}+2 d_{1 i} \omega_{i} s+\omega_{i}^{2}}\right. \\
& \frac{\alpha_{2 i} s}{s^{2}+2 d_{2 i} \omega_{i} s+\omega_{i}^{2}}, \ldots, \\
& \left.\frac{\alpha_{m i} s}{s^{2}+2 d_{m i} \omega_{i} s+\omega_{i}^{2}}\right)
\end{aligned}
$$

where, in both cases

$$
\alpha_{q i} \geq 0, \quad i=1,2 \ldots, N ; \quad q=1,2, \ldots, m .
$$

and

$$
\text { and } \sum_{i=1}^{N} \alpha_{q i}=1, \quad q=1,2, \ldots, m .
$$

It can be verified that both $J_{a}(s)$ and $J_{b}(s)$ are strictly proper WSPR systems. That is $J_{a}(j \omega)+J_{a}(j \omega)^{*}>0$ and $J_{b}(j \omega)+$ $J_{b}(j \omega)^{*}>0$ for all $\omega \in(-\infty, \infty)$. Hence, the resulting admittances will guarantee closed-loop stability of the system.

Corresponding to $J_{a}(s)$ and $J_{b}(s)$, the expressions for $Y_{a}(s)$ and $Y_{b}(s)$ can be determined as

$$
\begin{aligned}
Y_{a}(s)= & \operatorname{diag}\left(\frac{\sum_{i=1}^{N} \frac{\alpha_{1 i} \omega_{i}^{2}}{s^{2}+2 d_{1 i} \omega_{i} s+\omega_{i}^{2}}}{1-\sum_{i=1}^{N} \frac{\alpha_{1 i} \omega_{i}^{2}}{s^{2}+2 d_{1 i} \omega_{i} s+\omega_{i}^{2}}}\right. \\
& \frac{\sum_{i=1}^{N} \frac{\alpha_{2 i} \omega_{i}^{2}}{s^{2}+2 d_{2 i} \omega_{i} s+\omega_{i}^{2}}}{1-\sum_{i=1}^{N} \frac{\alpha_{2 i} \omega_{i}^{2}}{s^{2}+2 d_{2 i} \omega_{i} s+\omega_{i}^{2}}}, \ldots, \\
& \left.\frac{\sum_{i=1}^{N} \frac{\alpha_{m i} \omega_{i}^{2}}{s^{2}+2 d_{m i} \omega_{i} s+\omega_{i}^{2}}}{1-\sum_{i=1}^{N} \frac{\alpha_{m i} \omega_{i}^{2}}{s^{2}+2 d_{m i} \omega_{i} s+\omega_{i}^{2}}}\right) \Lambda^{-1} s
\end{aligned}
$$

and

$$
\begin{aligned}
Y_{b}(s)= & \operatorname{diag}\left(\frac{\sum_{i=1}^{N} \frac{\alpha_{1 i}\left(2 d_{1 i} \omega_{i} s+\omega_{i}^{2}\right)}{s^{2}+2 d_{1 i} \omega_{i} s+\omega_{i}^{2}}}{1-\sum_{i=1}^{N} \frac{\alpha_{1 i}\left(2 d_{1 i} \omega_{i} s+\omega_{i}^{2}\right)}{s^{2}+2 d_{1 i} \omega_{i} s+\omega_{i}^{2}}}\right. \\
& \frac{\sum_{i=1}^{N} \frac{\alpha_{2 i}\left(2 d_{2 i} \omega_{i} s+\omega_{i}^{2}\right)}{s^{2}+2 d_{2 i} \omega_{i} s+\omega_{i}^{2}}}{1-\sum_{i=1}^{N} \frac{\alpha_{2 i}\left(2 d_{2 i} \omega_{i} s+\omega_{i}^{2}\right)}{s^{2}+2 d_{2 i} \omega_{i} s+\omega_{i}^{2}}}, \ldots, \\
& \left.\frac{\sum_{i=1}^{N} \frac{\alpha_{m i}\left(2 d_{m i} \omega_{i} s+\omega_{i}^{2}\right)}{s^{2}+2 d_{m i} \omega_{i} s+\omega_{i}^{2}}}{1-\sum_{i=1}^{N} \frac{\alpha_{m i}\left(2 d_{m i} \omega_{i} s+\omega_{i}^{2}\right)}{s^{2}+2 d_{m i} \omega_{i} s+\omega_{i}^{2}}}\right) \Lambda^{-1} s .
\end{aligned}
$$

One of the interesting properties of the above admittance transfer functions is that over a specific bandwidth, one has the option of choosing to control only those modes that are of importance. This is reflected in the constraint on parameters $\alpha_{q i}$ in (16). This is in contrast to control-design methodologies such as LQG and $\mathcal{H}_{\infty}$, where the controller tends to have equal dimension to that of the system that is being controlled.

A further property of the controllers $Y_{a}$ and $Y_{b}$ is that in the presence of out of bandwidth modes of the base structure, they do not cause instabilities. The spillover effect [29] and [30] is a serious cause of concern in control design for flexible structures. Often, a feedback controller is designed using a model of the structure that contains a limited number of modes. Once the controller is implemented on the full order system, the presence of uncontrolled high-frequency modes may destabilize the closed-loop system, or severely deteriorate the performance. Considering the discussion in Section III, it should be clear that such a problem can not happen if the above procedure is followed. Stability of the shunted system with the above class of shunts is guaranteed. Furthermore, due to their highly localized nature, these shunts have the additional property that their effect on the out of bandwidth modes of the system is minimal, hence minimizing the spillover effect. 


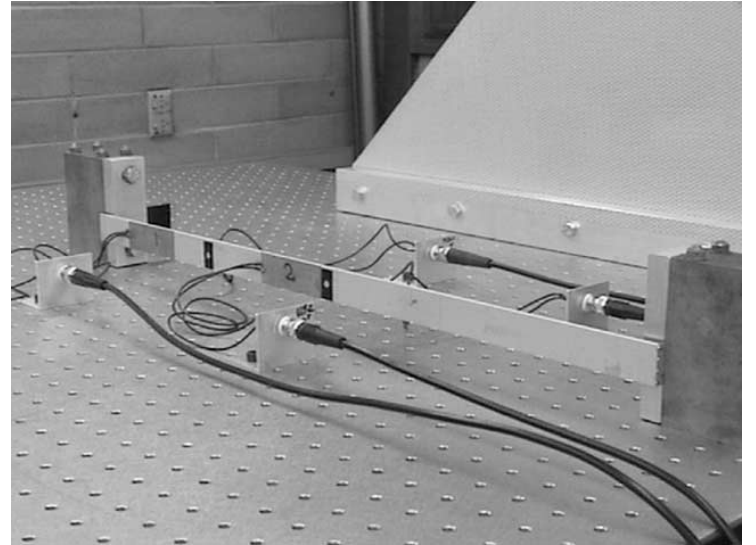

Fig. 6. Experimental beam apparatus.

Now, it is straightforward, but tedious, to verify that both $Y_{a}(s)$ and $Y_{b}(s)$ are SPR transfer functions. Therefore, they can be realized by passive circuit components; i.e., resistors, inductors, and capacitors. Given that both $Y_{a}(s)$ and $Y_{b}(s)$ have decentralized structures, effectively, each piezoelectric transducer is shunted by an independent admittance. However, it is not clear how such a network may be obtained as standard synthesis techniques result in realizations that require Gyrators and op-amps. To this end, it should be pointed out that even if passive realizations for (18) and (19) are found, in practice, such an implementation is likely to be impractical. Given that often low-frequency modes of a structure are targeted for shunt damping, the required inductors may be excessively large, in the order of several hundred to several thousand henries. A practical way of implementing $Y_{a}$ and $Y_{b}$ is to use the synthetic admittance circuit as described in [31], [19] or the alternative, and more effective method explained in Section V-C.

\section{EXPERIMENTAL RESULTS}

To validate the proposed concepts, experiments were carried out on a piezoelectric laminated beam at the Laboratory for Dynamics and Control of Smart Structures, ${ }^{1}$ at the University of Newcastle.

\section{A. Experimental Setup}

The test structure is a uniform aluminum beam with rectangular cross section and experimentally pinned boundary conditions. Two pairs of collocated piezoelectric patches are attached symmetrically to either side of the structure as shown in Figs. 6 and 7. Piezoelectric transducers used in our experiments are PIC1512 piezoelectric patches. Details of the beam and PIC151 piezoelectric patches are listed in Tables I and II.

The first pair of piezoelectric patches is placed close to one of the pinned ends of the beam, while the other pair is closer to the beam's center. Mode shapes of a simply-supported beam are sinusoidal functions [21]. Consequently, the first pair will be more effective in controlling the first vibration mode of the beam, while the second mode is better controlled by the second pair. One of the main advantages of multivariable shunt damping, as evident from this application, is that if one transducer does not

\footnotetext{
${ }^{1}$ http://rumi.newcastle.edu.au/

${ }^{2}$ These patches are manufactured by Polytec PI Ceramics.
}

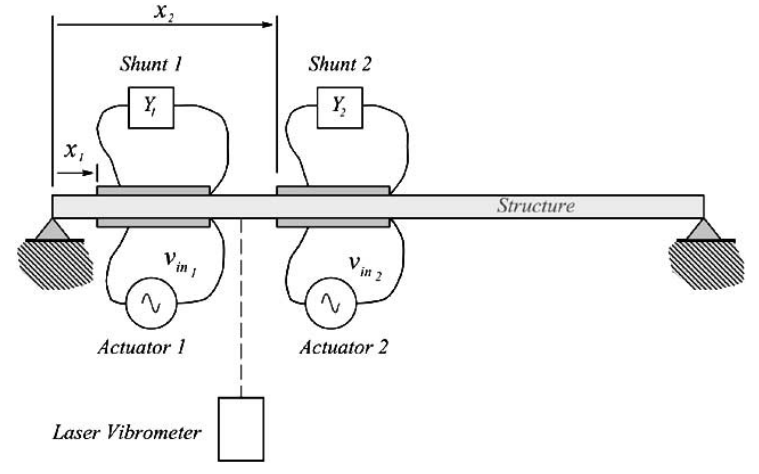

Fig. 7. Beam apparatus.

TABLE I

PARAMETERS OF THE SIMPLY-SUPPORTED BEAM

\begin{tabular}{c|c|c}
\hline Name & Symbol & Unit \\
\hline \hline Length & $L$ & $0.6 \mathrm{~m}$ \\
\hline Width & $w$ & $0.025 \mathrm{~m}$ \\
\hline Thickness & $h$ & $0.004 \mathrm{~m}$ \\
\hline Young's modulus & $E$ & $65 \times 10^{9} \mathrm{~N} / \mathrm{m}^{2}$ \\
\hline Poisson's ratio & $\nu$ & 0.3 \\
\hline Mass / unit area & $\rho$ & $10.6 \mathrm{~kg} / \mathrm{m}^{2}$ \\
\hline
\end{tabular}

TABLE II

PIC151 PieZoelectric PATCh PARAMETERS

\begin{tabular}{c|c|c}
\hline Name & Symbol & Unit \\
\hline \hline Location $x$-direction & $x_{1}$ & $0.050 \mathrm{~m}$ \\
\hline Location $x$-direction & $x_{2}$ & $0.240 \mathrm{~m}$ \\
\hline Length & $L_{p}$ & $0.0724 \mathrm{~m}$ \\
\hline Thickness & $h_{p}$ & $0.00191 \mathrm{~m}$ \\
\hline Width & $w_{p}$ & $0.025 \mathrm{~m}$ \\
\hline Capacitance & $C_{p}$ & $471 \times 10^{-9} \mathrm{~F}$ \\
\hline Young's modulus & $E_{p}$ & $62 \times 10^{9} \mathrm{~N} / \mathrm{m}^{2}$ \\
\hline Poisson's ratio & $\nu_{p}$ & 0.3 \\
\hline Strain Constant & $d_{31}$ & $-320 \times 10^{-12} \mathrm{~m} / \mathrm{V}$ \\
\hline Electromechanical coupling factor & $k_{31}$ & 0.44 \\
\hline Stress constant / voltage coefficient & $g_{31}$ & $-9.5 \times 10^{-3} \mathrm{~V} \mathrm{~m} / \mathrm{N}$ \\
\hline
\end{tabular}

offer enough authority over a specific mode, another transducer may enable the designer to control that mode more effectively.

\section{B. System Identification}

The first step in the analysis involves procuring a model for the transfer function matrix $G_{v v}(s)$. This will allow us to simulate the effect of an attached piezoelectric shunt on the transfer function from the applied actuator voltages $V_{\text {in }}(s)$, to the generated piezoelectric shunt layer voltages $V_{p}(s)$. These variables are internal and cannot be measured directly whilst an impedance is attached to the shunting layer. We will also consider the transfer function from the applied actuator voltages $V_{\text {in }}(s)$, to the structural deflection at a point $D(x, s)$. This point is chosen such that the first three modes of the beam are observable at that location. In the case where there are two actuators and two sensors, we require a model with two inputs and three outputs

$$
\left[\begin{array}{c}
V_{p}(s) \\
D(x, s)
\end{array}\right]=G_{p}(s) V_{\text {in }}(s)
$$

where $G_{p}(j \omega) \in \mathbf{C}^{3 \times 2}$ is the open-loop plant transfer function matrix. 

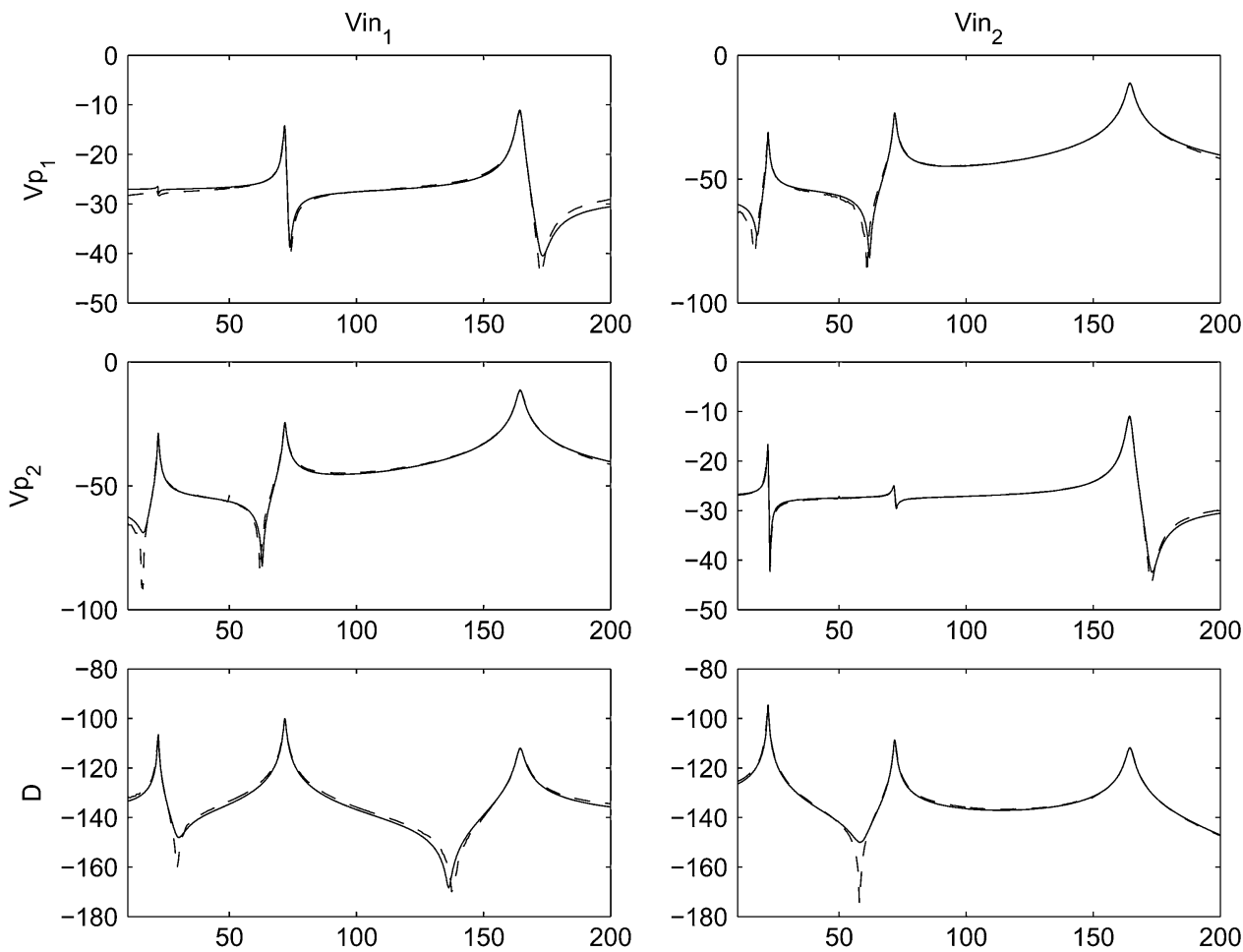

Fig. 8. Experimental data (--) and identified model $(-)$. Horizontal axes are in hertz, vertical axes in decibels. All the voltages are measured in volts. The displacement is measured in meters.

Modeling of piezoelectric laminate structures is generally accomplished in the literature by means of either analytic modeling, finite element analysis, or system identification. Analytic modeling, typically involving the assumed modes approach [21], requires distinct models for both structural dynamics and piezoelectric transducers [32]. Detailed information relating to the structural and piezoelectric physical properties is required. Practical application typically involves the use of experimental data and nonlinear optimization to identify unknown parameters such as resonance frequencies, piezoelectric coupling coefficients, and modal amplitudes. Another popular technique is that of the FE analysis [33]. This is an approximate method that results in high order spatially discrete models. If the dynamics of sensors and actuators are known, an integrated model can be cast in state-space form, facilitating design and analysis of passive shunt and feedback control systems [34]. As with the modal analysis procedure, FE models are usually tuned using experimental data [35].

System identification can be employed to procure a composite structural-piezoelectric model directly from experimental data. Although the field of system identification is extremely diverse [36], the range of possibilities is significantly reduced if we restrict ourselves to techniques capable of identifying multiple-input-multiple-output systems not requiring an explicit model parameterization or a nonlinear optimization, two rather undesirable traits. The majority of the residue comprises the so-called subspace class of system identification algorithms. Such methods identify state-space models by exploiting geometric properties of the input and output sequences. Methods exist for both time- and frequency-domain data, see [37] for a summary of time-domain methods. Frequency-domain

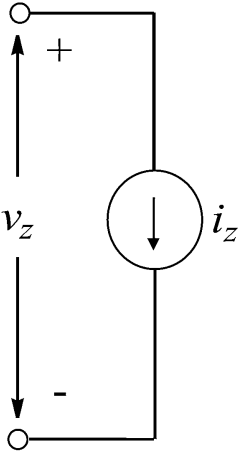

Fig. 9. Functionality of the synthetic impedance.

subspace methods have proved extremely effective in identifying high order resonant systems [24], [38]. In this paper, we employ the algorithm of Van Overschee and De Moor [39], a continuous-time frequency-domain subspace method $^{3}$ to identify a model of the system. Almost all available frequency-domain subspace algorithms will directly identify only discrete-time systems. This may be undesirable (as in our case) if the underlying system is continuous. The inconvenience is tolerated due to practical numerical advantages resulting from working with the orthonormal basis formed by the powers of $e^{j \omega}$. The algorithm [39] avoids the usual problems associated with the ill conditioned basis formed by the powers of $s$.

\footnotetext{
${ }^{3}$ A Matlab implementation of this algorithm is freely available by contacting the corresponding author or by visiting http://rumi.newcastle.edu.au.
} 


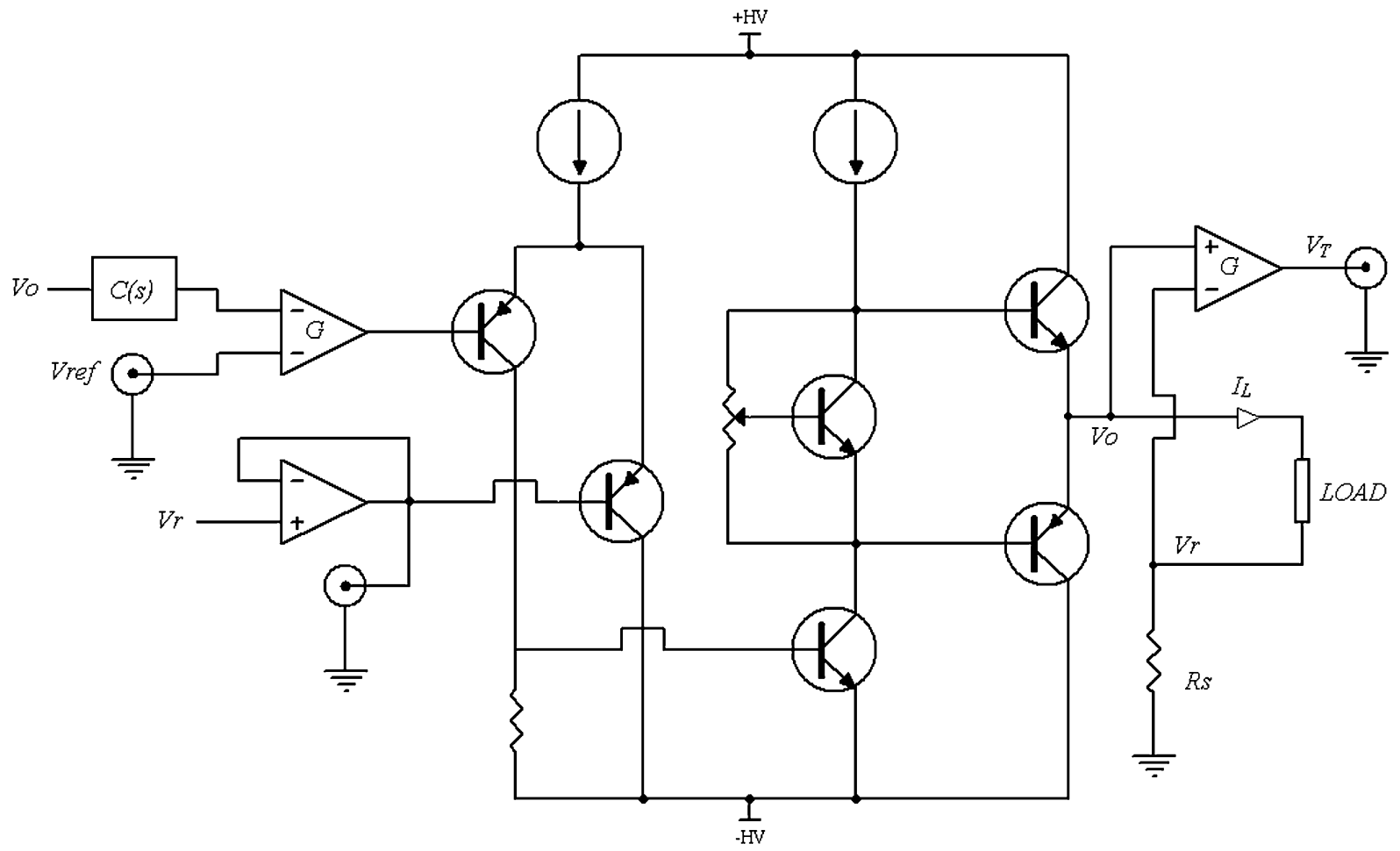

Fig. 10. Compliance controlled high-voltage current amplifier.

\section{Implementation of a Multiport Synthetic Admittance}

We now discuss the experiment performed to obtain a statespace representation of $G_{p}(s)$. Referring to Fig. 7, a prefiltered periodic chirp was applied to each actuating layer in succession. The resulting open circuit piezoelectric voltages and displacements were recorded using a dSPACE ds 1103 rapid prototyping system. The chirp prefiltering was performed by an FIR filter designed to reduce the power of the excitation in bands enclosing the resonance frequencies of the structure. This reduces the dynamic range and "flattens out" the power-spectral density and signal-to-noise ratio versus frequency at the outputs. An estimate for $G_{p}(j \omega)$ was then obtained using the empirical transfer function estimate [36]. The magnitude frequency response of $G_{p}(j \omega)$ is plotted in Fig. 8.340 frequency samples from 0 to 200 $\mathrm{Hz}$ were used to identify a sixth-order state model for $G_{p}(s)$. The magnitude frequency response of the model is overlain on the experimental data in Fig. 8.

References [19] and [31] introduce the synthetic admittance as a means for implementation of piezoelectric shunt damping circuits. The functionality is shown in Fig. 9. The voltage controlled current source $i_{z}$ is set as the output of a transfer function whose input is the voltage $v_{z}$ measured across the terminals, i.e., $I_{z}(s)=Y(s) V_{z}(s)$. The resulting impedance as seen from the terminals is $(1 / Y(s))$.

In this paper, an advanced technique for implementing piezoelectric shunt damping circuits is presented. The currentfeedback synthetic admittance (CFSA) allows highly accurate control and instrumentation of the terminal voltage and current. This device is suitable for multiport impedance synthesis, has a wide bandwidth ranging from millihertz to hundreds of kilohertz, and is suitable for high power operation (hundreds of watts continuous). No high-voltage opamps are required in this design.
The overall functionality is similar to that shown in Fig. 9. There are two main components, the current amplifier, and the admittance transfer function. As in [19], an analog filter or DSP system is used to implement the admittance transfer function. This controls the relationship between the measured terminal voltage and applied current. A discussion on the advantages of using such a technique is provided in [40].

A simplified circuit diagram of the current amplifier is shown in Fig. 10. The depicted current sources are used for static biasing and can be implemented using a resistor, or for increased open-loop gain and power-supply rejection [41], a transistor. The symbol $G$ denotes a standard low-gain differential amplifier.

Neglecting the compliance controller $C(s)$, a high-gain negative feedback loop ensures the applied reference voltage $V_{\text {ref }}$ appears across the sensing resistor $R_{s}$, i.e., the current through the load $I_{L}$ is set by $\left(V_{\text {ref }} / R_{s}\right)$. To reconfigure the circuit as a charge amplifier, the resistor $R_{s}$ is replaced by a capacitor. Charge/current sources have shown promise for driving piezoelectric loads as the function relating charge or current to strain exhibits less nonlinearity than the voltage driven equivalent [42]. Poor driver performance at low frequencies has limited the practical exploitation of this phenomena. Small but inevitable voltage and current offsets in a charge amplifier have the effect of charging up the load capacitance. Consequently, the uncontrolled output voltage drifts, reducing the compliance range, and eventually clipping the output voltage. In combat, a large parallel resistance is added to reduce the dc magnitude of the load impedance. Depending on the necessary size of the resistor (lower to compensate for larger offset currents), high-pass dynamics are added to the current amplifier, distorting the low-frequency magnitude response and introducing phase delay. Not only is the low-frequency response degraded, but the user may be impervious to 


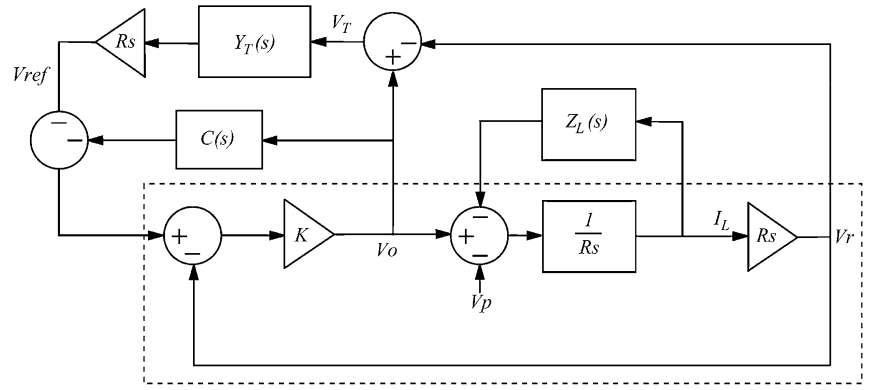

Fig. 11. System diagram of the current feedback synthetic impedance.

the degradation as the measured current is no longer that flowing through the load. By taking a multiobjective control approach, we propose a superior technique to compensate for low-frequency offset currents.

To fully understand the problem, consider the system diagram shown in Fig. 11. A synthetic admittance incorporating a current feedback amplifier (shown in the dashed rectangle) is attached to a load with an internal source $V_{p}$. The diagram relates the dynamics of the admittance transfer function $Y(s)$, the compliance controller $C(s)$, and the load impedance $Z_{L}(s)$ to the terminal voltage $V_{T}$, the compliance voltage $V_{o}$, and the load current $I_{L}$. In analogy to offset current compensation by parallel resistance, choosing $C(s)$ as a simple attenuator introduces a first order high pass filter into the current amplifier. To our advantage, we are now able to instrument the actual load current. By using a slightly more complicated compliance controller $C(s)$, we are able to completely eliminate residual compliance offsets and obtain a low-frequency bandwidth in the millihertz range. A simple attenuating integrator $C(s)=(1 / \alpha s)$ [41] will completely reject any source of dc offset current in the circuit, e.g., amplifier offsets, offsets in the reference voltage, offsets caused by asymmetric supply voltages, and digital-analog conveter offset errors if the transfer function $Y(s)$ is implemented using a digital signal processor. Neglecting dielectric loss in the load, the time constant of the system is controlled by the parameter $\alpha$. The only penalty for setting an extremely low high-pass cut off is the slow transient response to a step disturbance or at start up. The transient limitation of control systems with integral regulation is well understood and treated in any introductory text on control design. Low-frequency compliance feedback can also be used for electrical prestressing of piezoelectric actuators. Many piezoelectric actuators must only be used in mechanical compression. By adding an offset to the compliance feedback loop, an actuator can be driven by current or charge with a constant dc voltage offset across the load. This has the effect of altering the dc mechanical operating point of the device and making it suitable for bipolar applications such as active vibration control.

A photo of the experimental current amplifier is shown in Fig. 12.

\section{Implementing the Admittance Transfer Function}

On first inspection, the admittance structures (18) and (19) may appear difficult to implement by means of either analog or digital signal processing. In fact the reverse is true, the transfer function can be represented as a simple block diagram com-

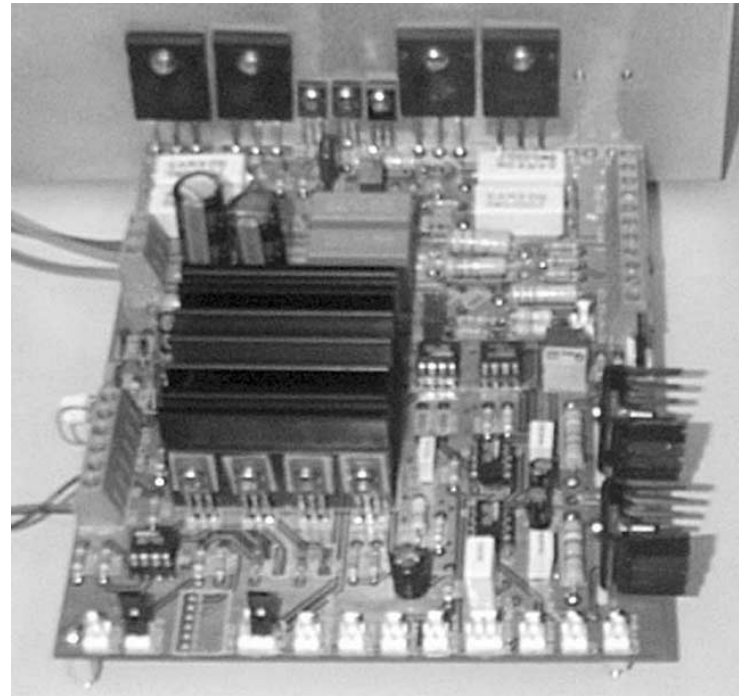

Fig. 12. Experimental current source.

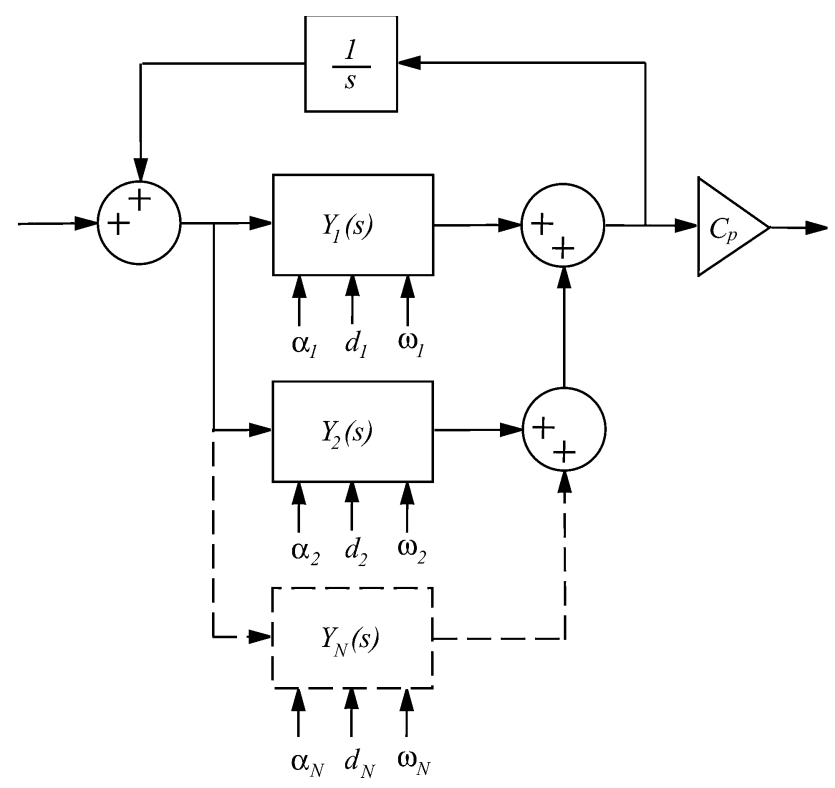

Fig. 13. System diagram of (18) or (19).

TABLE III

ADMITTANCE PARAMETERS

\begin{tabular}{|c|c|}
\hline Parameter & Value $(\mathrm{Hz})$ \\
\hline \hline$\omega_{1,2}$ & 71.7 \\
\hline$\omega_{1,3}$ & 161.6 \\
\hline$\omega_{2,1}$ & 22.14 \\
\hline$\omega_{2,3}$ & 167.9 \\
\hline
\end{tabular}

\begin{tabular}{|c|c|}
\hline Parameter & Value \\
\hline \hline$d_{1,2}$ & 0.021 \\
\hline$d_{1,3}$ & 0.024 \\
\hline$d_{2,1}$ & 0.025 \\
\hline$d_{2,3}$ & 0.023 \\
\hline \hline$\alpha_{1,2}$ & 0.5 \\
\hline$\alpha_{1,3}$ & 0.5 \\
\hline$\alpha_{2,1}$ & 0.5 \\
\hline$\alpha_{2,3}$ & 0.5 \\
\hline
\end{tabular}

posed of second order subsystems. Consider the admittance required for a single piezoelectric transducer using the controller

$$
Y_{a}(s)=\frac{\sum_{i=1}^{N} \frac{\alpha_{i} \omega_{i}^{2}}{s^{2}+2 d_{i} \omega_{i} s+\omega_{i}^{2}}}{1-\sum_{i=1}^{N} \frac{\alpha_{i} \omega_{i}^{2}}{s^{2}+2 d_{i} \omega_{i} s+\omega_{i}^{2}}} C_{p} s .
$$



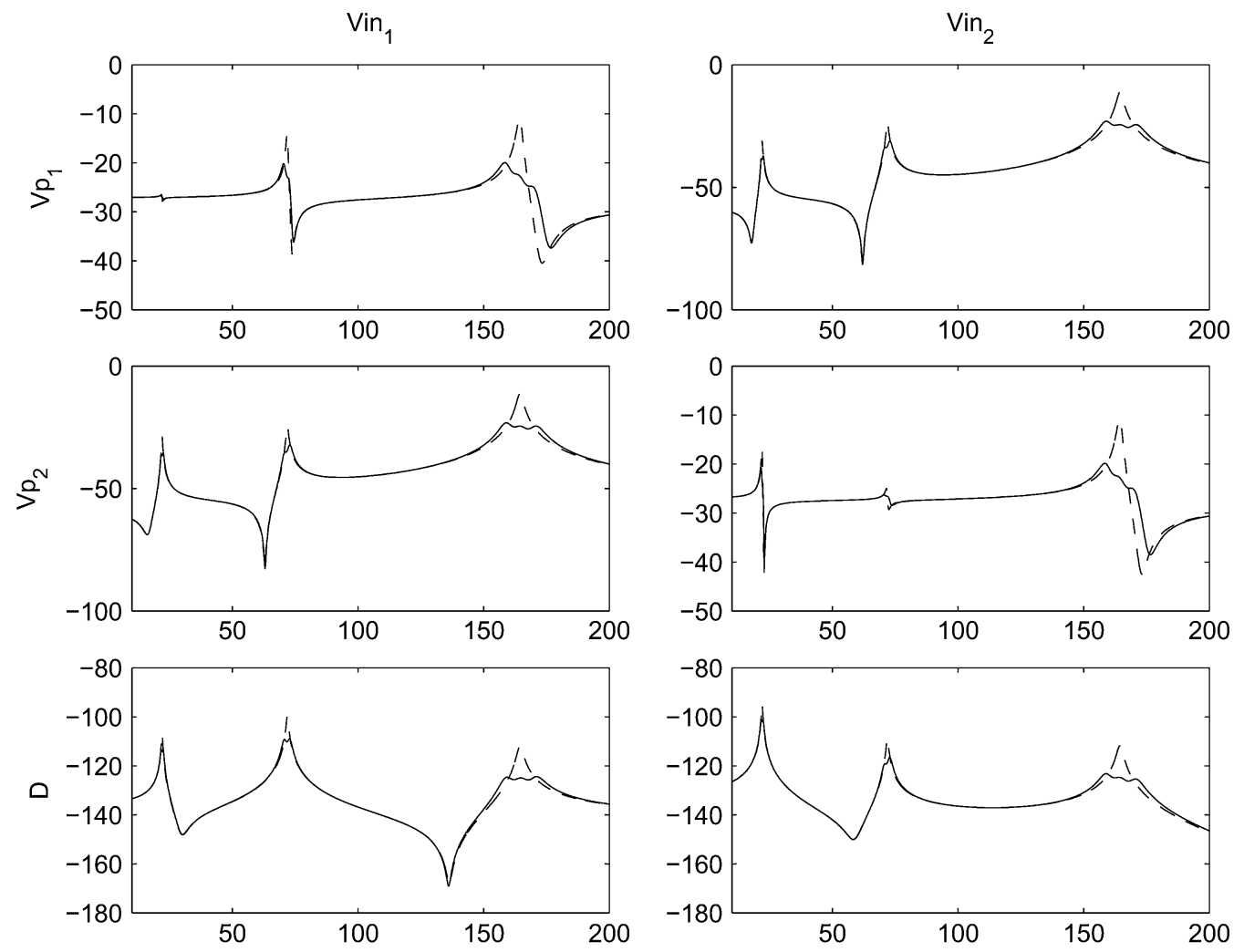

Fig. 14. Simulated open-loop (--) and closed-loop (-) magnitude frequency response. Horizontal axes are in hertz, vertical axes in decibels. All the voltages are measured in volts. The displacement is measured in meters.

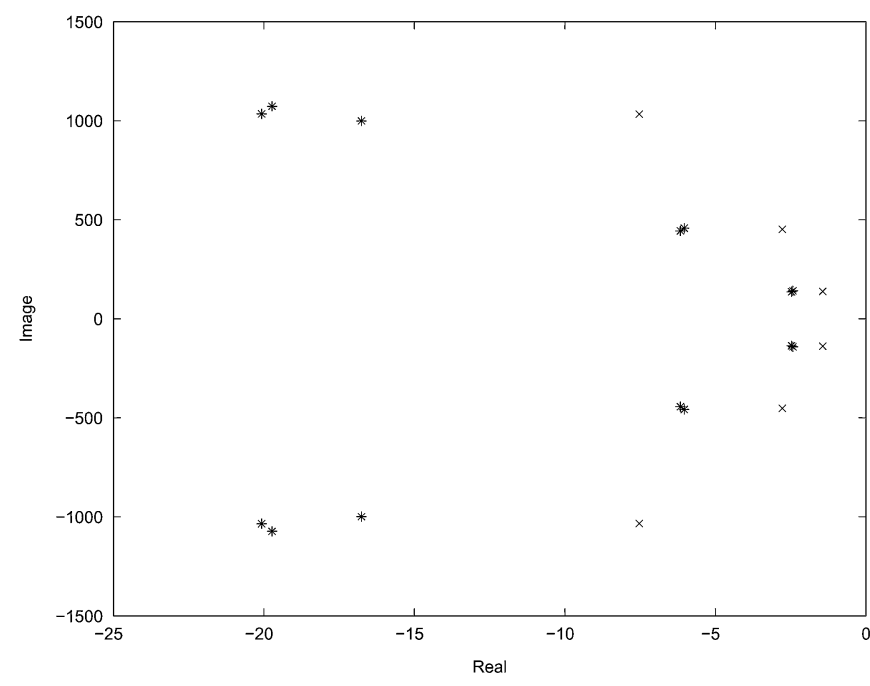

Fig. 15. Simulated open $(x)$ and closed-loop $(*)$ pole locations.

The structure (21) is shown diagrammatically in Fig. 13. Each subsystem $Y_{i}\left(s, \alpha_{i}, d_{i}, \omega_{i}\right)$, parameterized for ease of online tuning, can be implemented by an analog state variable filter [41], or internally in a DSP algorithm. For digital implementation, each subsystem is most easily parameterized in state-space form. For example

$$
Y_{i}\left(s, \alpha_{i}, d_{i}, \omega_{i}\right)=\frac{y_{i}}{u}=\frac{\alpha_{i} \omega_{i}^{2} s}{s^{2}+2 d_{i} \omega_{i} s+\omega_{i}^{2}}
$$
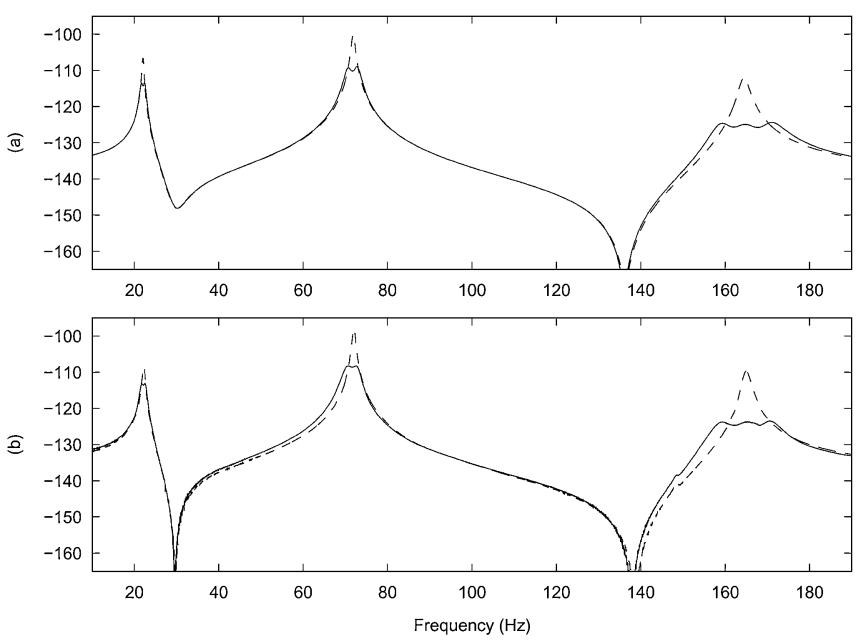

Fig. 16. (a) Simulated and (b) experimental open-loop (--) and closed-loop (一) frequency responses from $V_{\mathrm{in}_{1}}$ (volts) to the displacement measured at $x=0.17 \mathrm{~m}$ in decibels.

where

$$
\begin{aligned}
\dot{x} & =\left[\begin{array}{cc}
0 & 1 \\
-\omega_{i}^{2} & -2 d_{i} \omega_{i}^{2}
\end{array}\right] x+\left[\begin{array}{l}
0 \\
1
\end{array}\right] u \\
y_{i} & =\left[\begin{array}{ll}
0 & \alpha_{i} \omega_{i}^{2}
\end{array}\right] x .
\end{aligned}
$$

\section{E. Results}

In the experiments, one of the actuating piezoelectric transducers was used to disturb the structure. The two transducers on 

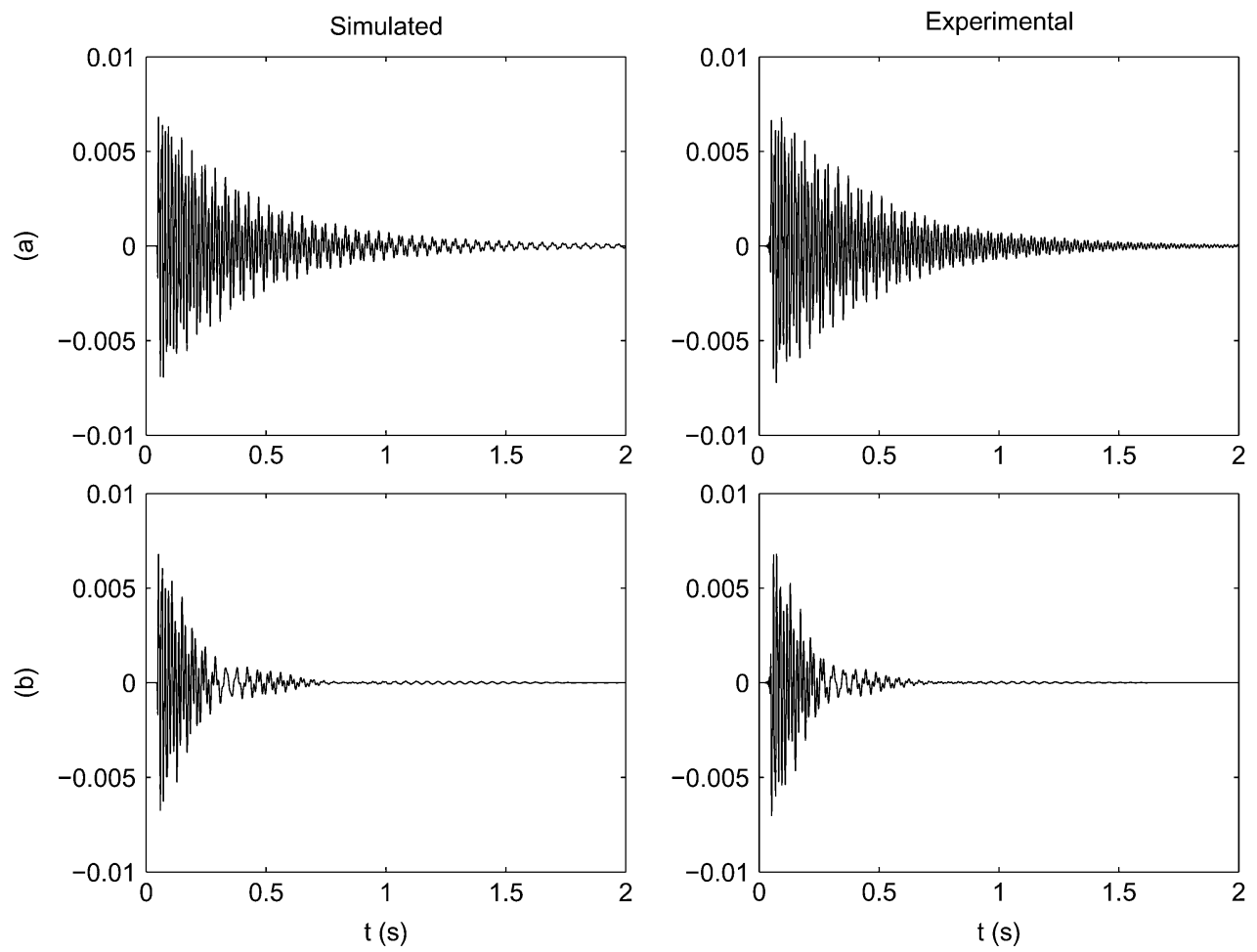

Fig. 17. (a) Open-loop and (b) closed-loop displacement response at $x=0.17 \mathrm{~m}$ to a lowpass filtered step input applied to $V_{\text {in }_{1}}$. The displacement is measured in meters.

the other side of the beam were shunted with resonant impedances to attenuate the vibrations generated in the beam. Using the structure $Y_{a}(s)$ (18), shunt circuits were applied to both of the piezoelectric transducers. Specifically, $Y_{1}(s)$ that was shunted to the first piezoelectric transducer and tuned to control the second and third modes, and $Y_{2}(s)$ that was shunted to the second piezoelectric transducer and tuned to control the first and third modes. Admittance parameters are shown in Table III. Parameters $\alpha_{1,2}, \alpha_{1,3}, \alpha_{2,1}$ and $\alpha_{2,3}$ are set equal to 0.5 to satisfy condition (17) and to put equal emphasis on every mode. The rest of the parameters are determined via the solution to an optimization problem.

The admittance has a diagonal structure

$$
Y_{a}(s)=\operatorname{diag}\left(Y_{1}(s), Y_{2}(s)\right)
$$

where

$Y_{1}(s)=$

$\left(\frac{\alpha_{1,2} \omega_{1,2}^{2}}{s^{2}+2 d_{1,2} \omega_{1,2} s+\omega_{1,2}^{2}}+\frac{\alpha_{1,3} \omega_{1,3}^{2}}{s^{2}+2 d_{1,3} \omega_{1,3} s+\omega_{1,3}^{2}}\right)$

$1-\left(\frac{\alpha_{1,2} \omega_{1,2}^{2}}{s^{2}+2 d_{1,2} \omega_{1,2} s+\omega_{1,2}^{2}}+\frac{\alpha_{1,3} \omega_{1,3}^{2}}{s^{2}+2 d_{1,3} \omega_{1,3} s+\omega_{1,3}^{2}}\right) C_{p} s$

and

$Y_{2}(s)=$

$\left(\frac{\alpha_{2,1} \omega_{1,1}^{2}}{s^{2}+2 d_{2,1} \omega_{1,1} s+\omega_{1,1}^{2}}+\frac{\alpha_{2,3} \omega_{1,3}^{2}}{s^{2}+2 d_{2,3} \omega_{1,3} s+\omega_{1,3}^{2}}\right)$

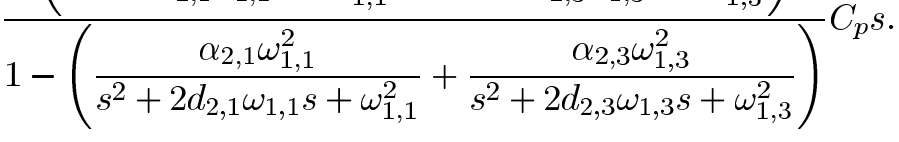

Fig. 14 compares the simulated frequency response of the unshunted system with that of the shunted system. The figure is associated with a $2 \times 3$ system whose inputs are the voltages applied to the two actuating piezoelectric patches, and whose outputs are the induced piezoelectric voltages and the displacement measurement at $x=0.17 \mathrm{~m}$. The displacement measurements were obtained using a PSV300 laser scanning vibrometer.

Fig. 15 demonstrates the effect of the proposed admittance structure. It can be observed that by shunting the two piezoelectric patches with the proposed admittances the closed-loop poles of the system have been pushed further into the left half of the complex plane. We notice that both transducers are used to dampen the third mode, while the first two modes are damped using the second, and first transducers respectively. This is due to the location at which the two patches are mounted on the beam. The first patch offers little authority over the first mode of the beam, while the second patch displays a similar lack of authority over the second mode. Both transducers however, are effectively reducing vibration corresponding to the third mode of the structure.

In experiments, variables internal to the piezoelectric transducers are not directly measurable. Therefore, it was not possible to generate experimental results corresponding to all entries of the transfer function matrix displayed in Fig. 14. However, as the displacement could be measured, results were obtained by applying a disturbance voltage to the first piezoelectric transducer and measuring the resulting displacement. The corresponding transfer functions are plotted in Fig. 16. It can be observed that the experimental results closely match the simulations. Experimental results show a considerable attenuation of the resonant peaks; $5 \mathrm{~dB}$ for the first mode, $10.5 \mathrm{~dB}$ for the second mode, and $14.4 \mathrm{~dB}$ for the third mode. 
To examine the time-domain performance of the damped system, a $200-\mathrm{Hz}$ low-pass-filtered step was applied to $V_{i n_{1}}$. The simulated and experimental displacement responses measured at $x=0.17 \mathrm{~m}$ are plotted in Fig. 17. Note that the response is dominated by the first mode of vibration. This is a result of the lower damping achieved for this mode, and the comparatively greater low-frequency Fourier components contained in a step function.

\section{CONCLUSION}

It was demonstrated that the problem of piezoelectric shunt damping with several piezoelectric transducers and a multiinput impedance is equivalent to a feedback control problem for a square plant. The controller itself was shown to be inside an inner feedback loop. A parameterization of stabilizing controllers/electrical shunts was introduced. Two decentralized shunts with favorable damping properties were proposed and their effectiveness in reducing structural vibrations was experimentally verified.

\section{REFERENCES}

[1] A. Baz and S. Poh, "Performance of an active control system with piezoelectric actuators," J. Sound Vibr., vol. 126, no. 2, pp. 327-343, 1988.

[2] T. Bailey and J. E. Hubbard, "distributed piezoelectric-polymer active vibration control of a cantilever beam," J. Guid. Control Dyn., vol. 8, no. 5, pp. 605-611, 1985.

[3] C. C. Sung, V. V. Varadan, X. Q. Bao, and V. K. Varadan, "Active control of torsional vibration using piezoceramic sensors and actuators," in Proc. AIAA/ASME/ASCE/AHS/ASC 31st Structures Structural Dynamics and Matrials Conf., 1990, pp. 2317-2322.

[4] J. Pan, C. H. Hansen, and S. D. Snyder, "A study of the response of a simply supported beam to excitation by a piezoelectric actuator," in Proc. Conf. Recent Advances in Active Control of Sound and Vibration, Blacksburg, VA, 1991, pp. 39-49.

[5] D. J. Inman and E. Garcia, "Smart structures for vibration suppression," in Proc. 4th Int. Conf. Recent Advances in Structural Dynamics, Southampton, U.K., July 15-18, 1991.

[6] S. S. Ge, T. H. Lee, and J. Q. Gong, "A robust distributed controller of a single-link scara/cartesian smart materials robot," Mechatron., vol. 9, pp. 65-93, 1999.

[7] N. W. Hagood and A. von Flotow, "Damping of structural vibrations with piezoelectric materials and passive electrical networks," J. Sound Vibr., vol. 146, no. 2, pp. 243-268, 1991.

[8] S. Y. Wu, "Method for multiple mode shunt damping of structural vibration using a single PZT transducer," in Proc. SPIE Symp. Smart Structures and Materials-Smart Structures and Intelligent Systems, Mar. 1998, pp. 159-167.

[9] J. J. Hollkamp, "Multimodal passive vibration suppression with piezoelectric materials and resonant shunts," J. Intell. Mater. Syst. Struc., vol. $5,1994$.

[10] S. Behrens and S. O. R. Moheimani, "Current flowing multiple mode piezoelectric shunt dampener," in Proc. SPIE Symp.Smart Structures and Materials-Damping and Isolation, vol. 4697, San Diego, CA, 2002, pp. 217-226.

[11] N. W. Hagood and E. F. Crawley, "Experimetnal investigation of passive enhancement of damping for space structures," AIAA J. Guid. Navigation Control, vol. 14, no. 6, pp. 1100-1109, 1991.

[12] S. Y. Wu, "Multiple PZT transducer implemented with multiple-mode piezoelectric shunt for passive vibration damping," in Proc. SPIE Symp. Smart Structures and Materials-Passive Damping and Isolation, Mar. 1999, pp. 112-122.

[13] S. J. Kim, C. H. Han, and C. Y. Yun, "Improvement of aeroelastic stability of hingeless helicopter rotor blade by passive piezoelectric damping," in Proc. SPIE Conf. Passive Damping and Isolation, Newport Beach, CA, Mar. 1999, pp. 131-141.
[14] E. Bianchini and R. Spangler, "The use of piezoelectric devices to control snowboard vibrations," in Proc. SPIE Conf. Integrated Systems, vol. 3329, 1998, pp. 106-114.

[15] P. Vallone, "High-performance piezo-based self-sensor for structural vibration control," in Proc. SPIE Conf. Smart Structures and Intelligent Systems, vol. 2443, 1995, pp. 643-655.

[16] J. B. Aldrich, N. W. Hagood, A. von Flotow, and D. W. Vos, "Design of passive piezoelectric damping for space structures," in Proc. SPIE Conf. Smart Structures and Intelligent Systems, vol. 1917, 1993, pp. 629-705.

[17] O. Thorp, M. Ruzzene, and A. Baz, "Attenuation and localization of wave propagation in rods with periodic shunted piezoelectric patches," Smart Mater. Struct., no. 10, pp. 979-989, 2001.

[18] R. H. S. Riodan, "Simulated inductors using differential amplifiers," Electron. Lett., vol. 3, no. 2, pp. 50-51, 1967.

[19] A. J. Fleming, S. Behrens, and S. O. R. Moheimani, "Optimization and implementation of multimode piezoelectric shunt damping systems," IEEE/ASME Trans. Mechatron., vol. 7, pp. 87-94, Mar. 2002.

[20] D. Halim and S. O. R. Moheimani, "Spatial resonant control of flexible structures-Application to a piezoelectric laminate beam," IEEE Trans. Contr. Syst. Technol., vol. 9, pp. 37-53, Jan. 2001.

[21] L. Meirovitch, Elements of Vibration Analysis, 2nd ed, Sydney, Australia: McGraw-Hill, 1986.

[22] P. C. Hughes, "Space structure vibration modes: How many exist? which ones are important?," IEEE Contr. Syst. Mag., vol. 7, pp. 22-28, Feb. 1987.

[23] T. McKelvey, H. Ackay, and L. Ljung, "Subspace-based multivariable system identification from frequency-response data," IEEE Trans. Automat. Contr., vol. 41, pp. 960-979, July 1996.

[24] T. McKelvey, A. J. Fleming, and S. O. R. Moheimani, "Subspace-based system identification for an acoustic enclosure," Tran. ASME Vibr. Acoust., vol. 124, no. 3, pp. 414-419, 2002.

[25] M. Morari and E. Zafiriou, Robust Process Control. Englewood Cliffs, NJ: Prentice-Hall, 1989.

[26] J. M. Maciejowski, Multivariable Feedback Design. Reading, MA: Addison-Wesley, 1989.

[27] S. M. Joshi and S. Gupta, "On a clss of marginally stable positive-real systems," IEEE Trans. Automat.Contr., vol. 41, pp. 152-155, Jan. 1996.

[28] J. T. Wen, "Time-domain and frequency-domain conditions for strict positive realness," IEEE Trans. Automat.Contr., vol. 33, pp. 988-992, Oct. 1998.

[29] M. J. Balas, "Active control of flexible systems," J. Optim. Theory Applicat., vol. 25, no. 3, pp. 415-436, 1978.

[30] — "Feedback control of flexible systems," IEEE Trans. Automat. Contr., vol. AC-23, pp. 673-679, Apr. 1978.

[31] A. J. Fleming, S. Behrens, and S. O. R. Moheimani, "Synthetic impedance for implementation of piezoelectric shunt damping circuits," Electron. Lett., vol. 36, no. 18, pp. 1525-1526, 2000.

[32] C. R. Fuller, S. J. Elliot, and P. A. Nelson, Active Control of Vibration. New York: Academic, 1996.

[33] R. D. Cook, Finite Element Modeling for Stress Analysis. New York: Wiley, 1995.

[34] Y.-H. Lim, V. V. Varadan, and V. K. Varadan, "Closed-loop finite element modeling of active/passive damping in structural vibration control," in Proc. SPIE Smart Materials and Structures, Mathematics and Control in Smart Structures, vol. 3039. San Diego, CA, Mar. 1997, pp. 113-124.

[35] N. M. Maia and J. M. Montalvao de Silva, Theoretical and Experimental Model Analysis. Hertfordshire, U.K.: Research Studies Press, 1997.

[36] L. Ljung, System Identification: Theory for the Users. Englewood Cliffs, NJ: Prentice-Hall, 1999.

[37] M. Viberg, "Subspace-based methods for the identification of linear time invariant systems," Automatica, vol. 31, no. 12, pp. 1835-1851, 1995.

[38] T. McKelvy, H. Akcay, and L. Ljung, "Subspace based multivariable system identification from frequency response data," IEEE Trans. Automat. Contr., vol. 41, pp. 960-978, July 1996.

[39] P. Van Overschee and B. De Moor, "Continuous-time frequency-domain subspace system identification," Signal Processing, vol. 52, pp. 179-194, 1996.

[40] A. J. Fleming, S. Behrens, and S. O. R. Moheimani. A new approach to piezoelectric shunt damping. presented at Proc. Int. Symp. Smart Structures and Microsystems

[41] P. Horowitz and W. Hill, The Art of Electronics. Cambridge, U.K.: Cambridge Univ. Press, 1980.

[42] C. Newcomb and I. Flinn, "Improving the linearity of piezoelectric actuators using charge feedback," Electron. Lett., vol. 18, no. 11, pp. 442-444, May 1982. 


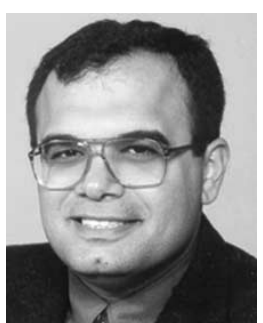

S. O. R. Moheimani (S'93-M'97-SM'00) was born in Shiraz, Iran, in 1967. He received the B.Sc. degree from Shiraz University, Shiraz, Iran, in 1990, and the M.Eng.Sc. and Ph.D. degrees from the University of New South Wales, Australia, in 1993 and 1996, respectively, all in electrical and electronics engineering.

In 1996, he was a Postdoctoral Research Fellow in the School of Electrical and Electronics Engineering, Australian Defence Force Academy, Canberra, Australia. In 1997, he joined The University of Newcastle, Newcastle, Australia, where he is currently an Associate Professor in the School of Electrical Engineering and Computer Science. His research interests include smart structures, mechatronics, control theory, and signal processing. He has over 100 journal and conference publications, and is coauthor of the research monograph Spatial Control of Vibration: Theory and Experiments (Singapore: World Scientific, 2003) and the Editor of the volume Perspectives in Robust Control (New York: Springer Verlag, 2001).

Dr. Moheimani is an Associate Editor for Control Engineering Practice, and International Journal of Control, Automation, and Systems. He has served on the editorial boards of several international conferences, and is the Chairman of International Program Committee for the 3rd IFAC Symposium on Mechatronic Systems, to be held in Sydney, Australia, in September 2004. He is a member of the IFAC Technical Committee on Mechatronic Systems.

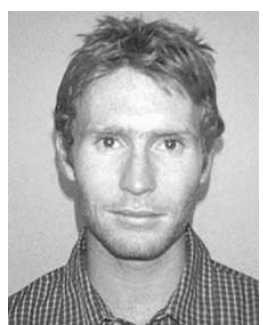

Andrew J. Fleming (M'00) was born in Dingwall, Scotland, in 1977. He received the B.E. (Elec.) and $\mathrm{Ph} . \mathrm{D}$. degrees from The University of Newcastle, Newcastle Australia, in 2000, and 2004, respectively.

$\mathrm{He}$ is a member of the Laboratory for Dynamics Control of Smart Structures. His research involves acoustic noise control of ducts; and passive, semi-active and self-sensing vibration control of piezoelectric laminates.

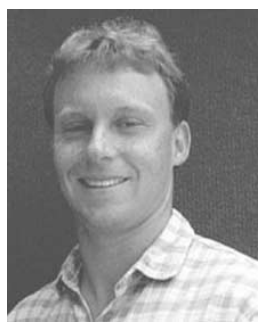

Sam Behrens was born in Sydney, Australia, in 1975 He received the B.E. (Mech.) and the M.E. degrees, in 1999 and 2001, respectively, from The University of Newcastle, Newcastle, Australia, where he is currently working toward the Ph.D. degree in the School of Electrical Engineering and Computer Science.

His research interests include passive, semi-active, and self-sensing vibration control systems. 